\title{
TWO QUESTIONS ON SCALAR-REFLEXIVE RINGS
}

\author{
NICOLE SNASHALL
}

(Communicated by Louis J. Ratliff, Jr.)

\begin{abstract}
A module $M$ over a commutative ring $R$ with unity is reflexive if the only $R$-endomorphisms of $M$ leaving invariant every submodule of $M$ are the scalar multiplications by elements of $R$. A commutative ring $R$ is scalarreflexive if every finitely generated $R$-module is reflexive. A local version of scalar-reflexivity is introduced, and it is shown that every locally scalar-reflexive ring is scalar-reflexive. An example is given of a scalar-reflexive domain that is not $h$-local. This answers a question posed by Hadwin and Kerr. Theorem 7 gives eight equivalent conditions on an $h$-local domain for it to be scalarreflexive, thus classifying the scalar-reflexive $h$-local domains.
\end{abstract}

A module $M$ over a commutative ring $R$ with unity is said to be reflexive if the only $R$-endomorphisms of $M$ leaving invariant every submodule of $M$ are the left scalar multiplications by elements of $R$. In [4], Hadwin and Kerr defined a commutative ring $R$ to be scalar-reflexive if every finitely generated $R$-module is reflexive. Throughout this paper all rings are commutative with unity. This paper considers two questions of Hadwin and Kerr on scalarreflexive rings.

Hadwin and Kerr ask in [3, p. 7] whether the property of being scalar-reflexive is preserved under localisations. They characterised the local scalar-reflexive rings in [4, Theorem 6], showing that a local ring is scalar-reflexive if and only if it is an almost maximal valuation ring (see Proposition 1). This result of Hadwin and Kerr motivates Definition 2, where a ring is defined to be locally scalar-reflexive if every localisation at a maximal ideal is scalar-reflexive. The first theorem in this paper proves that every locally scalar-reflexive ring is scalarreflexive. This result is given in Theorem 4 and provides a converse to the question raised by Hadwin and Kerr.

A second question of Hadwin and Kerr concerns the characterisation of the scalar-reflexive domains and they ask in $[4$, p. 318] whether every scalar-reflexive domain is an $h$-local domain. Matlis defined an $h$-local domain in $[5, \S 8]$ to be a domain such that every nonzero prime ideal is contained in a unique maximal ideal, and every nonzero element is contained in only finitely many maximal ideals. Example 5 provides an example of a scalar-reflexive domain that is not $h$-local, thus answering this second question in the negative.

Received by the editors February 1, 1991 and, in revised form, April 17, 1991.

1991 Mathematics Subject Classification. Primary 13F05; Secondary 13C99, 16A65.

The author was supported by an SERC grant. 
Although the scalar-reflexive domains are as yet unknown, the scalar-reflexive $h$-local domains may be characterised in a variety of ways. Theorem 7 gives eight equivalent conditions on an $h$-local domain for it to be scalar-reflexive. One of these properties is that every 2-generated torsion module is a direct sum of cyclic modules. This links the study of alglat and reflexivity with the structure and decomposition of modules. (An $R$-module $T$ is torsion if, for all $t \in T$, $\operatorname{Ann}(t) \neq 0$.) Theorem 7 also shows that an $h$-local domain is scalar-reflexive if and only if it is locally scalar-reflexive. This provides a partial converse to Theorem 4.

\section{DEFINITIONS AND NOTATION}

A module that is reflexive in the sense of the definition given above was called scalar-reflexive by Hadwin and Kerr in [3]. The terminology used here follows the more general notion of reflexivity given by Fuller, Nicholson, and Watters in [1]. For a bimodule ${ }_{R} M_{\Delta}$ where $R$ and $\Delta$ are rings with unity (not necessarily commutative), they define alglat ${ }_{R} M_{\Delta}$ to be the ring of all $\Delta$-endomorphisms of $M$ leaving invariant every $R$-submodule of $M$. The bimodule ${ }_{R} M_{\Delta}$ is then said to be reflexive if the elements of alglat $_{R} M_{\Delta}$ are precisely the left scalar multiplications by elements of $R$. This agrees with the definition above where $\Delta=R$, a commutative ring with unity. In this case alglat $_{R} M_{R}=\{\phi \in$ End $M_{R} \mid \phi m \in R m$ for all $\left.m \in M\right\}$. Let $\lambda$ be the map $\lambda: R \rightarrow \operatorname{alglat}_{R} M_{R}$ where $\lambda(r): m \mapsto r m$. Then it is always true that $\lambda(R) \subseteq$ alglat $_{R} M_{R}$. The bimodule is reflexive when there is equality, that is, $\lambda(R)=$ alglat $_{R} M_{R}$. Where it will not cause confusion, alglat $M$ is written for alglat ${ }_{R} M_{R}$.

In [3], Hadwin and Kerr defined a ring $R$ to be strongly scalar-reflexive if every $R$-module is reflexive and strictly scalar-reflexive if every finitely generated $R$-module is reflexive. They omitted the word "strictly" in [4], giving the definition of scalar-reflexive above, since they completely characterised all strongly scalar-reflexive rings in that paper. The notation "scalar-reflexive" is used throughout this paper, even when referring to [3].

For any $S$-module $M$, Ann $M$ is taken to be the annihilator of $M$ in the ring $S$. In particular, for finitely generated modules, Ann $R x$ denotes the annihilator of the $R$-module $R x$ in $R$, whereas Ann $R_{M} y$ is used for the annihilator of the $R_{M}$-module $R_{M} y$ in $R_{M}$.

A valuation ring $R$ is maximal if every system of pairwise soluble congruences of the form $\left\{x \equiv x_{\alpha} \bmod I_{\alpha}\right\}$ has a simultaneous solution in $R$, where $x_{\alpha} \in R, I_{\alpha}$ is an ideal of $R$, and $\alpha$ is in some index set $J$. A valuation ring is almost maximal if the above congruences have a simultaneous solution whenever $\bigcap_{\alpha \in J} I_{\alpha} \neq 0$.

A ring is an FGC ring if every finitely generated module over the ring is a direct sum of cyclic submodules ([9]). It was noted by Hadwin and Kerr in [3, p. 3] that a finite direct sum of cyclic modules is reflexive. Thus the FGC rings provide examples of scalar-reflexive rings [3, Proposition 4].

\section{SCALAR-REFLEXIVE RINGS AND LOCALISATIONS}

The first proposition characterises the local scalar-reflexive rings and was proved by Hadwin and Kerr in [4] with the equivalence of property (4) noted in $[4$, p. 316]. 
Proposition 1 [4, Theorem 6]. Let $R$ be a local ring. Then the following are equivalent:

(1) $R$ is scalar-reflexive;

(2) $R$ is an FGC ring;

(3) $R$ is an almost maximal valuation ring;

(4) every 2-generated $R$-module is reflexive.

This result can be used to provide information on rings whose localisations are scalar-reflexive and motivates the following definition.

Definition 2. A ring is locally scalar-reflexive if every localisation at a maximal ideal is scalar-reflexive, equivalently, if every localisation at a prime ideal is scalar-reflexive.

To prove this equivalence, suppose that every localisation at a maximal ideal is scalar-reflexive. Let $P$ be a prime ideal of $R$ and let $M$ be a maximal ideal of $R$ containing $P$. Then $R_{P} \cong\left(R_{M}\right)_{P_{M}}$. Since $R_{M}$ is scalar-reflexive, $R_{M}$ is an almost maximal valuation ring (Proposition 1). Then, using [2, Lemma 2], $\left(R_{M}\right)_{P_{M}}$ is also an almost maximal valuation ring. So $R_{P}$ is an almost maximal valuation ring, and hence is scalar-reflexive (Proposition 1). Thus every localisation at a prime ideal is scalar-reflexive.

The first question to be considered was raised by Hadwin and Kerr in [3, p. 7] and asks whether the class of scalar-reflexive rings is closed under localisations. A particular case of this, when the localisation are at the maximal ideals, asks whether every scalar-reflexive ring is locally scalar-reflexive. Theorem 4 is the main result of this section and proves a converse, showing that every locally scalar-reflexive ring is scalar-reflexive. However, the only known examples of scalar-reflexive rings are also locally scalar-reflexive. This leaves the original question open. 4.

The following lemma, whose proof is straightforward, is required in Theorem

Lemma 3. Let $R$ be a commutative ring with a maximal ideal $M$ and let $T$ be an $R$-module. The localisation $T_{M}$ is an $R_{M}$-module under the obvious product. Then the map $\alpha$ : alglat $T_{R} T_{R} \rightarrow$ alglat $T_{M}$ given by $\phi \mapsto \phi_{M}$, where $\phi_{M}: t / s \mapsto \phi t / s$, is a ring homomorphism.

Theorem 4. Every locally scalar-reflexive ring is scalar-reflexive.

Proof. Let $R$ be a locally scalar-reflexive ring, and let $\left\{M_{i} \mid i \in I\right\}$ be the set of all maximal ideals of $R$. Let $T=R x_{1}+R x_{2}+\cdots+R x_{n}$ be a finitely generated $R$-module, and let $\phi \in$ alglat $_{R} T_{R}$.

Let $M_{i}$ be any maximal ideal of $R$. Then there is a map $\phi_{M_{i}}$ in alglat $T_{M_{i}}$ given by $t / s \mapsto \phi t / s$ (Lemma 3 ). The $R_{M_{i}}$-module $T_{M_{i}}$ is finitely generated and is therefore reflexive (by hypothesis). So there is an element $a_{i} / u_{i}$ in $R_{M_{i}}$ with $\phi_{M_{i}}=\lambda\left(a_{i} / u_{i}\right)$. Then $\phi_{M_{i}}\left(x_{j} / 1\right)=a_{i} x_{j} / u_{i}=\phi x_{j} / 1$ for each $j=1, \ldots, n$, so there are elements $s_{i j} \in R \backslash M_{i}$ and $\left(a_{i} x_{j}-u_{i}\left(\phi x_{j}\right)\right) s_{i j}=0$. Let $s_{i}=\prod_{j=1}^{n} s_{i j}$ so then $s_{i} \notin M_{i}$. Then $\left(a_{i} x_{j}-u_{i}\left(\phi x_{j}\right)\right) s_{i}=0$ for all $j=1, \ldots, n$. So $u_{i}, s_{i} \notin M_{i}$ and, for $j=1, \ldots, n, u_{i} s_{i}\left(\phi x_{j}\right)=a_{i} s_{i} x_{j}$.

The sum $\sum_{i \in I} R u_{i} s_{i}=R$, for otherwise there is some maximal ideal $N$ with $\sum_{i \in I} R u_{i} s_{i} \subseteq N$. But $N=M_{k}$ for some $k \in I$ and $u_{k} s_{k} \notin M_{k}$ giving the required contradiction. So there is a finite subset $K$ of $I$ with $1=$ 
$\sum_{k \in K} r_{k} u_{k} s_{k}$ and $r_{k} \in R$. Then, for $j=1, \ldots, n, \phi x_{j}=\sum_{k \in K} r_{k} u_{k} s_{k}\left(\phi x_{j}\right)=$ $\sum_{k \in K} r_{k} a_{k} s_{k} x_{j}$. Let $r=\sum_{k \in K} r_{k} a_{k} s_{k}$ so that $\phi x_{j}=r x_{j}$ for $j=1, \ldots, n$ and $r \in R$. Then $\phi=\lambda(r)$ and so $T$ is reflexive. Hence $R$ is scalar-reflexive.

Theorem 4 also provides an extension of a result by Hadwin and Kerr. In [4, Theorem 10] they proved that, for an $h$-local domain $R$ with $R_{M}$ an almost maximal valuation ring for all maximal ideals $M$ of $R, R$ is scalar-reflexive. The hypotheses of this theorem may be rewritten, requiring $R$ to be an $h$-local domain which is locally scalar-reflexive. From Theorem 4 , it is clear that the condition that $R$ be an $h$-local domain is redundant.

\section{SCALAR-REFLEXIVE $h$-LOCAL DOMAINS}

Hadwin and Kerr ask in $[4$, p. 318$]$ whether every scalar-reflexive domain is $h$-local. This question is answered in the negative by Example 5, which uses rings of type $I$. In [7], Matlis defined a ring $R$ to be of type $I$ if $R$ is an integral domain with exactly two maximal ideals $M_{1}$ and $M_{2}$ such that $R_{M_{1}}$ and $R_{M_{2}}$ are maximal valuation rings and there is no nonzero prime ideal contained in $M_{1} \cap M_{2}$. An example by Osofsky of such a ring is included in [7].

The ring in Example 5 was given by Matlis in [8, Example 2] as an example of a ring which is the intersection of two dependent maximal valuation rings, but is not a ring of type $I$. This ring is now shown to be a locally scalar-reflexive (and hence scalar-reflexive) domain that is not an $h$-local domain.

Example 5. Let $A$ be a ring of type $I$ with two maximal ideals $M_{1}$ and $M_{2}$. Let $B$ be the field of fractions of $A$. Let $R$ be the ring of formal power series in an indeterminate $X$ with coefficients in $B$ but with constant term in $A$, so that $R=\left\{\sum_{0}^{\infty} b_{i} X^{i} \mid b_{0} \in A, b_{i} \in B\right.$ for $\left.i>0\right\}$. Then $R$ is a domain. Let $P$ be the prime ideal of $R$ consisting of power series with constant term $b_{0}=0$. Then $R$ has precisely two maximal ideals $N_{1}=M_{1}+P$ and $N_{2}=M_{2}+P$. The prime ideal $P$ satisfies $0 \neq P \subseteq N_{1} \cap N_{2}$. Thus $R$ is not an $h$-local domain.

The power series in the domain $R_{N_{1}}$ have constant term in $A_{M_{1}}$. Then $R_{N_{1}}$ is a valuation ring since $A_{M_{1}}$ is a valuation ring. It is known that $\left(R_{N_{1}}\right)_{P} \cong R_{P}$. Since $R_{P} \cong B[[X]]$, a maximal valuation ring, the ring $\left(R_{N_{1}}\right)_{P}$ is also a maximal valuation ring. The quotient ring $R_{N_{1}} / P$ is a maximal valuation ring too, being isomorphic to $A_{M_{1}}$. For a valuation domain $S$ and prime ideal $Q$ of $S$, $S$ is a maximal valuation ring if and only if both $S_{Q}$ and $S / Q$ are maximal valuation rings (a proof can be found in [8, Corollary 2]). Thus $R_{N_{1}}$ is a maximal valuation ring. Similarly $R_{N_{2}}$ is a maximal valuation ring. Thus $R$ is locally scalar-reflexive.

Hence $R$ is a scalar-reflexive domain that is not an $h$-local domain.

Having shown that not every scalar-reflexive domain is $h$-local, the problem of characterising the scalar-reflexive domains remains open. However, Theorem 7 gives a variety of equivalent characterisations of the scalar-reflexive $h$-local domains. The principal component of Theorem 7 is the proof that, for an $h$-local domain $R$ with every 2 -generated $R$-module reflexive, $R$ is locally scalar-reflexive. This result is included as Theorem 6 .

In order to prove Theorem 6, some properties of $h$-local domains from [6] are required. Matlis defined an ideal of an integral domain $R$ to be colocal if it is contained in only one maximal ideal of $R$. For an $h$-local domain $R$ with $I$, 
$J, I_{i}$ nonzero ideals of $R$ and $M, M_{i}$ maximal ideals of $R(i=1, \ldots, n)$, the following are properties of colocal ideals:

(i) if $I$ and $J$ are colocal in $M$ then $I \cap J$ is colocal in $M$;

(ii) if $I$ is colocal in $M$ and if $v \notin M$ then $I+R v=R$;

(iii) if $\bigcap_{i=1}^{n} I_{i}$ is a normal decomposition with $I_{i}$ colocal in $M_{i}$ then $I_{i}+$ $\bigcap_{i=2}^{n} I_{i}=R$

(iv) if $J=\bigcap_{i=1}^{n} I_{i}$ is a normal decomposition with $I_{i}$ colocal in $M_{i}$ and if $J \subseteq M$ then $M \in\left\{M_{1}, \ldots, M_{n}\right\}$.

Matlis proved in [6, Theorem 2.3] that a domain $R$ is an $h$-local domain if and only if every nonzero ideal of $R$ is a finite intersection of colocal ideals. A decomposition $I=\bigcap_{i=1}^{n} I_{i}$ into a finite intersection of ideals, where $I_{i}$ is colocal in the maximal ideal $M_{i}(i=1, \ldots, n)$, is said to be normal if $M_{j} \neq M_{k}$ for $j \neq k$. Thus (from (i)) every finite intersection of colocal ideals can be normalised.

Theorem 6. Let $R$ be an h-local domain. Then the following are equivalent:

(1) $R$ is locally scalar-reflexive;

(2) $R$ is scalar-reflexive;

(3) every 2-generated $R$-module is reflexive.

Proof. The implication (1) $\Rightarrow(2)$ is given in Theorem 4 and $(2) \Rightarrow(3)$ is trivial.

(3) $\Rightarrow(1)$. It is sufficient to show, for all maximal ideals $M$ of $R$, that every 2-generated $R_{M}$-module is reflexive. For then $R_{M}$ is scalar-reflexive for all maximal ideals $M$ (Proposition 1) and so $R$ is locally scalar-reflexive.

Let $M$ be a maximal ideal of $R$, and let $T=R_{M} x+R_{M} y$ be a 2-generated $R_{M}$-module. Suppose that both $R_{M} x$ and $R_{M} y$ are nonzero. For if not, then $T$ is cyclic and thus reflexive. The ring $R$ is a domain and so $R$ embeds in $R_{M}$ via $r \mapsto \frac{r}{1}$. For any element $t$ of $T$, there is a well-defined $R$-module structure on $T$ given by $r t:=\frac{r}{1} t$. There are two cases to consider.

Case 1: $T$ non-torsion. The local ring $R_{M}$ is a domain. It follows from [4, Proposition 7] that $T$ is reflexive.

Case 2: $T$ torsion. The first step is to find new generators $x^{\prime}$ and $y^{\prime}$ for $T$ so that the ideal $\operatorname{Ann}\left(R x^{\prime}+R y^{\prime}\right)$ is colocal in $M$. The construction begins by showing that the ideal Ann $R x$ is nonzero and is contained in $M$. The module $T$ is torsion so let $\frac{r}{u}$ be a nonzero element of $\operatorname{Ann}\left(R_{M} x\right)$. Then $r \neq 0, \frac{r}{1} \in \operatorname{Ann}\left(R_{M} x\right)$, and $r x=\frac{r}{1} x=0$. Thus Ann $R x \neq 0$. Suppose Ann $R x \nsubseteq M$, and let $s$ be an element in Ann $R x$ with $s \notin M$. Then, since $R_{M}=R_{M} \frac{1}{s}$, it follows that $R_{M} x=R_{M} s x=0$, a contradiction. Thus Ann $R x \subseteq M$.

So Ann $R x$ has a normal decomposition Ann $R x=\bigcap_{i=1}^{n} I_{i}$ with $I_{i}$ colocal in $M_{i}$. From above Ann $R x \subseteq M$ and so $M \in\left\{M_{1}, \ldots, M_{n}\right\}$. Suppose $M=M_{1}$. Since $\bigcap_{i=2}^{n} I_{i}+I_{1}=R$, there are elements $a$ in $\bigcap_{i=2}^{n} I_{i}$ and $b$ in $I_{1}$ with $1=a+b$. Then Ann $R a x=I_{1}$. For if $r \in \operatorname{Ann} R a x$, then $\operatorname{rax}=0$ and so $r a \in$ Ann $R x \subseteq I_{1}$. Thus $r=r a+r b \in I_{1}$. For the reverse inclusion let $r \in I_{1}$ so that $r a \in \bigcap_{i=1}^{n} I_{i}=$ Ann $R x$. Then $\operatorname{rax}=0$ and so $r \in$ Ann Rax. Thus Ann $\operatorname{Rax}=I_{1}$ which is colocal in $M_{1}=M$. It is also clear that $a$ is not an element of $M$ and so $R_{M} a x=R_{M} x$.

In the same way there is an element $c$ in $R$ and a colocal ideal $J$ of $M$ 
with $R_{M} c y=R_{M} y$ and Ann $R c y=J$. Let $x^{\prime}=a x$ and $y^{\prime}=c y$. Then $T=R_{M} x^{\prime}+R_{M} y^{\prime}$ and $\operatorname{Ann}\left(R x^{\prime}+R y^{\prime}\right)=\operatorname{Ann} R x^{\prime} \cap \operatorname{Ann} R y^{\prime}=I_{1} \cap J$, which is colocal in $M$. Thus $x^{\prime}$ and $y^{\prime}$ are the new generators.

The next step is to prove that $R t=R_{M} t$ for all $t$ in $T$. Let $t \in T$. Then clearly $R t \subseteq R_{M} t$. For the reverse inclusion let $\frac{r}{u} \in R_{M}$ so that $\frac{r}{u} t \in R_{M} t$. Since $\operatorname{Ann}\left(R x^{\prime}+R y^{\prime}\right)$ is colocal in $M$ and $u \notin M$, it follows that $R u+$ $\operatorname{Ann}\left(R x^{\prime}+R y^{\prime}\right)=R$. Thus there are elements $f$ in $R$ and $g$ in $\operatorname{Ann}\left(R x^{\prime}+R y^{\prime}\right)$ with $1=f u+g$. Then $\frac{1}{u}=\frac{f}{1}+\frac{g}{u}$ giving $\frac{r}{u} t=\frac{f r}{1} t+\frac{r}{u} g t$. But $g t=0$. (Write $t=\frac{d_{1}}{s_{1}} x^{\prime}+\frac{d_{2}}{s_{2}} y^{\prime}=\frac{1}{s_{1} s_{2}}\left(\frac{d_{1} s_{2}}{1} x^{\prime}+\frac{d_{2} s_{1}}{1} y^{\prime}\right)$. Thus $g t=\frac{g}{1} t=\frac{g}{s_{1} s_{2}}\left(d_{1} s_{2} x^{\prime}+d_{2} s_{1} y^{\prime}\right)=0$.) So $\frac{r}{u} t=\frac{f r}{1} t=f r t \in R t$. Thus $R_{M} t \subseteq R t$. Hence $R_{M} t=R t$ for all $t$ in $T$. In particular $T=R_{M} x^{\prime}+R_{M} y^{\prime}=R x^{\prime}+R y^{\prime}$ since $x^{\prime}$ and $y^{\prime}$ are in $T$.

The final step is to show that $T$ is a reflexive $R_{M}$-module. Considering $T$ as an $R$-module, $T=R x^{\prime}+R y^{\prime}$ is 2-generated and so is reflexive by hypothesis. Thus alglat $T_{R}=\lambda(R) \subseteq \lambda\left(R_{M}\right) \subseteq$ alglat $_{R_{M}} T_{R_{M}}$. But $R \subseteq R_{M}$ and so End $T_{R_{M}} \subseteq$ End $T_{R}$. Then alglat $R_{M} T_{R_{M}}=\left\{\phi \in\right.$ End $T_{R_{M}} \mid \phi t \in R_{M} t$ for all $t \in T\} \subseteq\left\{\phi \in\right.$ End $T_{R} \mid \phi t \in R_{M} t$ for all $\left.t \in T\right\}=\left\{\phi \in\right.$ End $T_{R} \mid \phi t \in R t$ for all $t \in T\}=$ alglat $_{R} T_{R}$. Thus alglat $R_{M} T_{R_{M}}=\lambda\left(R_{M}\right)$ and hence $T$ is a reflexive $R_{M}$-module.

Theorem 7 extends Theorem 6 and is the main result of this section, characterising the scalar-reflexive $h$-local domains.

Theorem 7. Let $R$ be an h-local domain. Then the following are equivalent:

(1) $R$ is scalar-reflexive;

(2) every finitely generated torsion $R$-module is reflexive;

(3) every finitely generated torsion $R$-module is a direct sum of cyclic modules;

(4) every 2-generated $R$-module is reflexive;

(5) every 2-generated torsion $R$-module is reflexive;

(6) every 2-generated torsion $R$-module is a direct sum of cyclic modules;

(7) $R$ is a Prüfer domain and $Q / R$ is injective, where $Q$ is the quotient field of $R$ and $Q / R$ is considered as an $R$-module;

(8) $R_{M}$ is an almost maximal valuation ring for every maximal ideal $M$ of $R$;

(9) $R$ is locally scalar-reflexive.

Proof. The proof of (1) $\Leftrightarrow(4) \Leftrightarrow(9)$ has already been given in Theorem 6 , and the results $(3) \Leftrightarrow(7) \Leftrightarrow(8)$ are proved by Matlis in [6, Theorem 5.7]. It follows from the definition of a locally scalar-reflexive ring and from Proposition 1 that $(8) \Leftrightarrow(9)$. The implications $(3) \Rightarrow(2) \Rightarrow(5)$ and $(3) \Rightarrow(6) \Rightarrow(5)$ are trivial. Finally the implication (5) $\Rightarrow(4)$ follows from [4, Proposition 7] since $R$ is a domain. Thus all nine properties are equivalent.

Remark. Using Theorem 6, it can be shown that (1) is a local property for $h$ local domains. Hence these properties are all local properties (since for any prime ideal $P$ of an $h$-local domain $R, R_{P}$ is a local domain and hence an $h$-local domain). 
Finally recall the result of Hadwin and Kerr [4, Theorem 10], discussed above. The equivalence of properties (1) and (8) of Theorem 7 for $h$-local domains gives a second generalisation of their result.

\section{ACKNOWLEDGMENTS}

I wish to express my thanks to my supervisor Dr. J. F. Watters for all his help and encouragement. This work forms part of my Ph.D. thesis at the University of Leicester.

\section{REFERENCES}

1. K. R. Fuller, W. K. Nicholson, and J. F. Watters, Reflexive bimodules, Canad. J. Math. 41 (1989), 592-611.

2. D. T. Gill, Almost maximal valuation rings, J. London Math. Soc. (2) 4 (1971), 140-146.

3. D. Hadwin and J. W. Kerr, Scalar-reflexive rings, Proc. Amer. Math. Soc. 103 (1988), 1-8.

4. __ Scalar-reflexive rings. II, J. Algebra 125 (1989), 311-319.

5. E. Matlis, Cotorsion modules, Mem. Amer. Math. Soc., vol. 49, Amer. Math. Soc., Providence, RI, 1964.

6. __ Decomposable modules, Trans. Amer. Math. Soc. 125 (1966), 147-179.

7. __ Rings of type I, J. Algebra 23 (1972), 76-87.

8. _ Rings with property D, Trans. Amer. Math. Soc. 170 (1972), 437-446.

9. T. S. Shores and R. Wiegand, Rings whose finitely generated modules are direct sums of cyclics, J. Algebra 32 (1974), 152-172.

Department of Mathematics, University of Leicester, Leicester, ENGland LE1 7RH 